\title{
OPENNESS OF NATURAL INFORMATION PROCESSING SYSTEMS AND SHORT-TERM AND LONG-TERM MEMORY
}

\author{
Vladimir Shumilov ${ }^{*}$, Vladimir Syryamkin \\ ${ }^{1}$ National Research Tomsk State University, 634050, Tomsk, Russia
}

\begin{abstract}
In this paper, various types of memory are considered. Development of the nervous system and the brain of organisms ensures expansion of species with more developed brain. Different characteristics of the brain zones have created different conditions for forming traces of events. The various traces of trace events formed in different parts of the brain provided a wider range of functions and brain responses, which contributed to the survival and expansion of species. The work describes the appearance of memory varieties and the causes of these varieties.
\end{abstract}

\section{The need for openness of self-organizing information processing systems}

Let us consider the philosophical aspect of the amount of information in the device being created (or in the person being brought up), which arises during the creation and education of the mind both artificial and natural. You can often hear statements as if reinforced by reasoning that no creator can create something more perfect than he himself is. This, ostensibly, follows from the statement that information does not arise from nothing. Therefore, a person or another creature cannot create something more perfect and more informed than a creator is. If a creator did not have information, where did it come from? This question arises when information is placed in the information storage medium of artificial intelligence (AI) directly during the process of its manufacturing.

The statement about impossibility would be legitimate if the person and the device being created or the creature being brought up (artificial intelligence or a child) were an isolated system. That is the person or the device being created or the creature being brought up could not use other sources of information, except for the information of the creator. In fact, the subject being created (or brought up) is comprehensively open, therefore, can receive and collect information from other sources than its creator or educator. Other teachers and educators, sources of concentrated information accumulated by other people, results from various means of information fixation and storage, and information carriers can act as third-party sources. They also include rock paintings, and books, various paper and electronic media, various artifacts created by other media, and various natural and social phenomena, and the primary sources of information (sources of relations, which are of

\footnotetext{
*Corresponding author: vnshumilov@rambler.ru
} 
interest to the observer). Therefore, because of the openness to other sources of information, the creature (trainee) can accumulate more information than the creator possesses and become more perfect than the creator (teacher) is; we can notice this very often. There examples of outstanding scientists, philosophers, public Figures, military leaders, and athletes, as well as examples at the family level because children are often smarter than parents are.

Similarly, the created artificial intelligence in order to surpass its creator must be capable of perceiving information not only from its creator, but also from the surrounding world. Therefore, it is necessary to create not the AI, which is filled with necessary information and is ready for immediate functioning, but a device (system) capable of training and self-training, that is a device capable of high-speed receiving and fixing information, which had not been embedded in it during the creation. A device should be able to use this information for functioning, rather than for simple storage of this information, which cannot be controlled entirely by the creators because of the enormous speed of information flow. It is almost impossible and economically inefficient to control completely the information flow received by the intellect developed because of their volume. Therefore, the real (not demonstrational) intellect (both artificial and natural) should be supplemented with information continuously during functioning.

Developed intellect regardless of whether it is natural or artificial, operates with a huge amount of information. In [1], researchers give an estimation of the information capacity of the human brain (no less than 300 terabytes) and the resulting rate of filling this capacity with information (on average, no less than 30,000 connections between neurons, or 60 kilobytes per second). Most likely, information is perceived by the human brain even faster, because the perception channels, for example, visual information channel (eyes) can skip information faster than the mentioned 60 kilobytes per second. Indeed, the human eye contains several tens of millions of photosensitive cells that can trigger several times per second.

\section{A mechanism for implementing the openness of information processing systems}

How can it be possible to record a large amount of information in the created artificial or born up natural intelligence, as well as the observed speed of perception and full processing of a powerful information flow throughout the life (the entire time of functioning) of the subject (or device with AI)? Such a system should be open to the perception of information in a variety of forms. The natural system that processes this powerful flow is being selforganized throughout the life, that is during the period of formation, growth of the organism, and in the adulthood. This system is formed by a simple cell division starting with one original (maternal) cell. We have proposed a purely local mechanism for selforganization of the information processing system in the organism. Processing consists in the passage of signals coming from the receptors along various paths through the brain structures to the effector (manipulators). Because of the fact that receptors require the expenditure of always-limited resources of the organism, first, receptors reacting to environmental influences dangerous to the organism were formed. Signals are generated only in receptors when the environment exposes them. When effectors receive signals that have passed through the structures of the brain, they perform some actions and carry out the organism's reaction to the irritating effect of the environment. If (successful) actions of the effectors result in stoppage of the irritating effect of the medium on the receptors, then the brain ceases to receive signals, that is the movement of signals through the brain and actions of the effectors cease. The system will go into hibernation until the next signal from the receptors arrives. 
When passing through the structures of the brain signals form traces of events that had given rise to these signals, that is they form new connections between neurons. These connections represent the organism's memory. They are associative, because they are formed exclusively (only) between two simultaneously excited neurons. Their association consists in the fact that the events that gave rise to the signals occurred about the same time. The connections accumulate the organism's experience. Subsequently, newly formed links along with the old ones ensure the forecast (prediction) of the arrival of dangerous events from the chains of events when one of the events in this chain is detected. Connections represent pathways for propagation of signals that have entered the brain. So, natural systems of information processing are organized extremely simply. Therefore, their selforganization is possible beginning with a single initial (mother) cell to a highly developed organism and a brain in it. Self-organization of the brain proceeds without a centralized assessment of the situation and control of the actions of neurons from the outside. Neurons functioning proceeds completely independently. They have no means of interacting with a distant or intimate environment, except for their entrances and exits.

Therefore, formation of links is carried out purely by a local mechanism. The weights of the connections are constantly changing. A continuous decrease in weights causes the process of forgetting.

\section{Short-term and long-term memory}

A brief preliminary examination of the functioning of the natural system of information processing shows the parameters of the memory of the organism, that is the parameters of the inter-neural connections, are determined by the elements of the mechanisms of fixing information (bonding) and by the rate of reduction of the weights of these links.

It becomes obvious that the properties of memory (inter-neuronal connections) depend on the parameters of the environment, in which these links reside, and on the parameters of the active elements (neurons), which form the bonds between themselves. The dependence of the memory decay on the environment, in which neurons and the connections between them are located (not the organism's environment), is that the connections, whatever their nature is, reduce their weights depending on the biological activity of the environment. In any case, the reduction in the weights of the connections will occur as a result of processes such as diffusion (resorption and decrease in the density of structures that form connections). The rate of processes that reduce the weights of connections will depend on the gradients of potentials of various types (electrical, chemical and others) in a given medium.

Just like memory fading, formation of connections and their initial weight depend on the environment and on the properties of the neurons that form the connections. Formation of connections depends on the characteristics of the medium. For example, it depends on the electrical strength of the surface separating the excited neurons as well as on the magnitude of potentials at the excited neurons. It depends on whether the potential difference between the inputs and outputs of the excited neurons exceeds the formation potential (FP) of the connection, and how much. First, FP depends, on the environment in which the neurons are formed. The potentials at the outputs and at the inputs of the excited neurons depend, first of all, on the structure and parameters of the neurons. These potentials also depend on the loads (on the number of associative connections for a given neuron).

It is clear that both the characteristics of the medium and the characteristics of the neurons (especially the magnitude of the loads (the number of associative connections)) will differ significantly in different areas of the brain. And these zonal differences will also have external manifestations: the ease of memorization and the various rates of forgetting. 
Manifestations of these differences are classified by psychologists as the existence of various types of memory [2].

We cannot control the area of our brain (memory), which participates in the reproduction of the image of an event. Therefore, based on statistics, a conclusion is made simply about the nature of memory: if it is a long-term or short-term, or some kind of intermediate. Psychologists identify almost dozens of memory types depending on the speed of forgetting the recorded events $[2,3]$.

Figure $1 a$ shows the curve of forgetting of the events (word reproduction) obtained experimentally. The resulting curve differs significantly from the usual type of curves in similar processes. In all processes, the measured quantities relating to flow and accumulation vary approximately exponentially (capacitor charge, potential equalization, the number of atoms that have not yet disintegrate up, and the level of the liquid flowing out of the capacitance. This nature of the change in quantities is because the rate of change of quantities is proportional to the difference between the magnitude and the limit, to which this quantity tends. What does it mean? Most likely, the result (the ability of human memory structures to reproduce event traces) is because it appears as the sum of several parallel processes and several indicators corresponding to these processes.
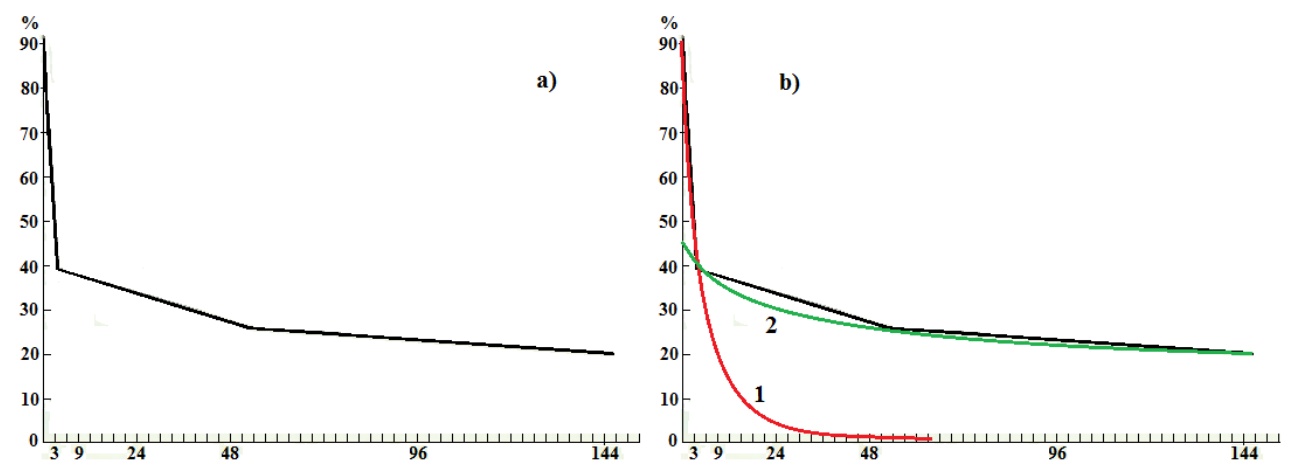

Fig. 1. The playback graph of the stored information.

Figure $1 b$ shows the representation of the experimental curve through two exponents. One of them falls very quickly (curve 1), while curve 2 falls relatively slowly. Therefore, the concepts of short-term and long-term memory were introduced into consideration. What is the reason for the presence of two types of memory? What is the mechanism of this phenomenon?

Here is a simplified scheme of the simplest natural information processing system (Figure $3 a$ ) and a slightly more complicated one (Figure $3 b$ ), in which connections were formed responsible for the reproduction of images of events that left traces (connections).

In the upper part a) of Figure 2 is a diagram of the simplest nervous information processing system consisting of one receptor, one effector, and the connection between them.

Figure $2 b$ shows a slightly more complicated system, which has the following distinctive features: 1) it reacts not to one, but to 3 stimuli, 2) it has 3 receptors and 3 effectors, and simple and direct (direct, without branching) connections between them. As a result of the increase in the number of connections in a limited volume, they begin to touch each other. The possibility of mutual influence between neighboring connections occurs because of formation of connections between the paths of signal transmission.

In the course of evolution, the nervous system is developing. An increasing number of input sensors (receptors) appears. And, correspondingly, the number of ways to promote the input signals from the receptors to the effectors is increasing. These paths necessarily 
influence each other, because they are in close proximity. Moreover, the number of effectors becomes much less than the number of receptors. Thus, the number of photosensitive elements (receptors) in the eye is estimated in tens of millions of elements. It is clear that the organism cannot have the same number of effectors reacting to the incoming stimuli of these receptors.

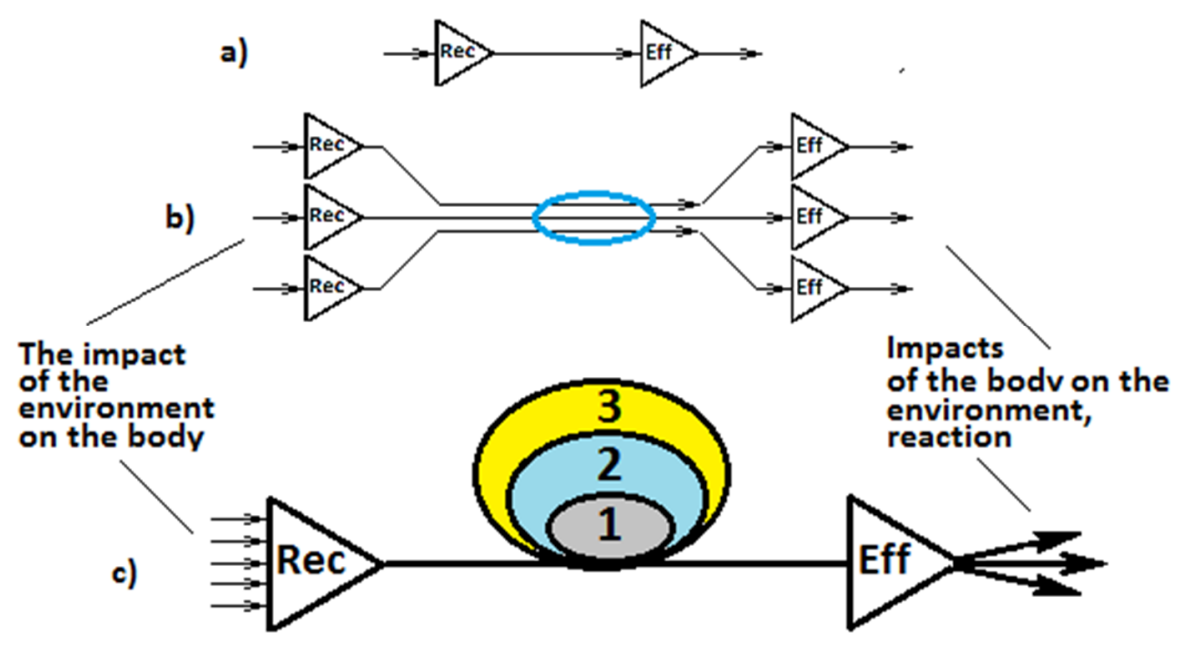

Fig. 2. Simplified diagram of the evolution of natural information processing systems.

So the signals from all the receptors are sent to the zone of their processing. These signals (afferent) after their processing (already efferent) pass through the existing paths to the corresponding effector. The actuation of the effectors carries out the organism's reaction to stimuli signals that had entered the brain through detectors. When a group of receptors and effectors are located remotely from the brain, afferent and efferent signals (centripetal and centrifugal) pass in close proximity to each other (in common channels) along the path from the place of generation to the place of effective response, where they can also interact. However, it is not so important for our problem.

Because the central part of the nervous system (the brain) was formed in the course of evolution for many generations, then its structures grew not at once but gradually. We can observe the consequences of this development in the brain structures of more developed animals (for example, fish, amphibians, birds, and mammals) [4, 5].

We need this observation to show that the signal pathways from the receptors to the effectors pass through the zones of the brain with different degrees of deepening into the brain and into its central zones of the brain, which have larger signal processing capabilities.

Inter-neural connections that conduct signals through different areas of the brain, of course, differ in their characteristics. In this case, we are interested in the ease of formation of inter-neural connections and the rate of their destruction and extinction (reducing their weights), and the speed of forgetting the information that gave rise to these connections.

Let us consider how the properties of memory can depend on the area of the brain, in which the connections were formed by the signals generated by the event of interest. For this, we refer to the mechanism of formation of connections between neurons [1]. Formation of connections between neurons occurs when neurons are in contact, and are both excited. If the potential difference between their points becomes higher than the potential of forming of the connection (PF), a new connection is formed. 
Subsequently, the formed links (traces of events) will conduct up signals through themselves based on this connection, thus forecasting the arrival of subsequent events. This ensures the possibility of evading the organism from the impact of predicted events.
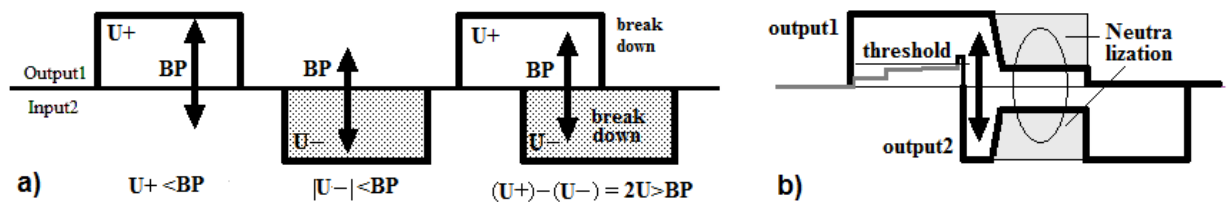

b)

Fig. 3. Conditions of formation of communication between neurons and compensation of potentials of excited neurons.

This Figure clearly shows how the parameters of neurons and their loads (the number of connections in neurons) affect formation of connections. Figure $3 a$ shows schematically the moment of formation of the connection between the output of one neuron and the input of another one. We can see that formation of a new connection depends on the magnitude of the potential generated by the neuron and on the electrical strength of the surface, which separates the neighboring excited neurons. Figure $3 b$ shows the compensation of the different polar potentials of the outputs and inputs of neurons with galvanic contacts (connections). The process of formation of connections between neurons is described in more detail in [1].

We note that the processes of formation of traces of events (formation of new links between excited neurons) and new signal paths (accumulation of experience) take place completely locally regardless of any external influences. This ensures parallelism and high speed of information processing in the brain. Information is fixed in the brain not in the storage mode (as simple placing of information for storage), but by means of changing the brain structures, which changes the possible paths of signals through the brain structures and the brain's reaction to future events. Continuous training and retraining of the brain in accordance with the environment occurs. These properties of the brain ensure its openness to the assimilation of information from a variety of sources throughout life.

According to the same principle, connections can be formed not only between natural biological neurons, but also between artificial neural-like elements in engineering devices created by engineers [6]. The efficiency of this principle of recording events in memory has been confirmed on the electronic model of the simplest nervous system created at Tomsk State University (TSU) [7].

In the course of evolution, the human brain has increased (through mutations of individual organisms during the process of generating new generations, survival and expansion of more viable organisms), because a larger brain contributed to a more successful survival and expansion of species. This growth of the brain most easily could occur because of the growth of new structures of the brain with the preservation of the already existing ones. Because formation of new areas of the brain occurred under slightly different conditions than of the earlier structures, the parameters of new neuronal structures differed somehow from the parameters of the earlier (ancient) structures. That is, new thresholds of neuron excitation, different potentials for formation of new connections, and other extinction rates of information links could have formed on new sites. It means that when the brain and cortex volume increased, regions with slightly different parameters of active and passive memory elements (neurons and connections between them) were formed in the new regions of the brain. These changes turned out to be useful and, therefore, became constant. 
Let us demonstrate the usefulness of forgetting in general, and different rates of forgetting information, which has different significance for survival of the organism. Forgetting gives an organism an opportunity to learn some reaction and do not loop on it. Without forgetting, the organism could not change its behavior in conditions of changes in environment, which occur constantly. Some changes are insignificant and information about these events does not penetrate deep into the brain, but some are very important and they cause with many associations. Therefore, they are fixed in different regions of the brain with different characteristics. For example, we are walking along a road covered with leaves; we see them and perceive them. However, there is no sense to keep in memory all the information about each of the leaf seen. Therefore, according to experimental data [8], information of this kind is stored for 20 seconds on the average. Similarly, when traveling along this road, we see all the defects on the road surface and even remember them all for a while. However, tomorrow we will be able to remember only the most dangerous and unusual defects and we will successfully forget all minor defects. Storage of such information in our memory does not make sense for our behavior.

Our brain acts rationally, it remembers important information and forgets the secondary one. In fact, the brain remembers everything. However, the rate of forgetting different types of information (the extinction of ties) is different. Therefore, psychologists talk about longterm and short-term memory; they experimentally measure the rates of forgetting [9]. The information values (remembered by subjects of an experiment) represented on graphs of psychologists cannot be represented as simple dependences of the type of declining exponents. However, they can be represented as a sum of simple dependencies. This indicates the placement of the same information in different zones, where the conditions for fixing information in the form of inter-neural connections and the rate of extinction of connections (connections weights reduction) differ.

How does the brain distinguish (with the declared locality of the mechanisms of memory), which traces of events should be placed in a more operative "short-term" memory, and what should be placed in a long-term memory? It turns out that there is no need to sort information when it arrives, it can be fixed simultaneously in many places. However, the more important is information, the more associative links it has. Therefore, a greater number of connections is formed for the same reason. In addition, new connections that are more persistent can be formed where the excitation of neurons (and connection formation) requires a larger signal provided by a large number of associative connections. In such zones of the brain, the formation of connections is heavier in comparison with the zones where connections with a high rate of extinction are formed, but the rate of extinction of the connections (information) in these zones is lower than in those zones where the connections are formed easily. If the information could have been fixed only in a zone with a high rate of forgetting (with a small number of associations), it effaced from the memory quickly. Perhaps this resource can be reused.

The shortest (instantaneous) memory is conditioned by the inertia of sensations received by the brain when the environment influence on the receptors. For example a bright flash of light remains in sensations up to a few seconds.

Next comes a little more long-term memory (perception), which is necessary for primary processing in the brain (to have time for perception). Not only sensations from the receptors, but also a certain number of connections from the information processing zones act as inputs. Perception allows you to embody sensations in images that are acceptable to the brain, it means that illuminated spots and points on the retina turn into signals indicating lines and certain silhouettes because of connections between neurons formed in the brain that has just started functioning. The brain is trained to act like this at the very beginning of its activity. In addition, there are also connections between things (events) felt by the young organism by various organs (receptors), what it sees with the eyes and feels with hands and 
a tongue. The results of perception move further along the brain as already formed images of a specific brain and can make traces not of signals from individual receptors but signals of image activation in subsequent parts of the brain.

Even further, (logically) from the signal input to the brain, a slightly more long-term (operational) memory is located; it has a large number of associative links. The signals in it remain active for a while, which helps to identify links between events separated by a certain period. However, connections formed in it quickly dissolve, and it is a useful property for the organism. The question that has not yet received an answer is very interesting for it: "Why the Miller's magic number is $7( \pm 2)$ (the number of elements held in this memory)? What is the mechanism for regulating the number of elements held?" [10].

The existence of images in this memory may be because traces of events in this memory fade very quickly. On the other hand, from the point of view of a possible biological realization Miller's number may be because the images of a certain number of events in a certain area of the brain (near the entrance) circulate until attenuation. This number cannot be more than a certain limit because of mutual suppression of signal flows through brain structures. From the point of view of functioning such circulation increases the possibility of formation of connections between neurons. Because the time intervals for the excitation of neurons are small, it is almost impossible to form associations between events separated by time intervals, more time is required to find the neuron in the excited state. While the time-extended activity of neuron chains contributes to formation of connections.

Further, specialized in perception and association areas of long-term memory come (motor, visual, auditory, mathematical, and others).

As a signal generated in the receptors moves, it meets different types of memory (including the operational one). This division has no clear boundaries. Such a progressively developed structure of the signal propagation pathways ensured detection and fixation of cause-effect relationships between events separated by sufficiently large (all `large) time intervals, much greater than the time of the complete cycle of excitation of one neuron. This gave the organisms greater chances of survival and expansion than detection of an associative link between events occurring simultaneously. Because of the more successful survival and expansion of the species with such brain structure, not only the population of the species but also the volumes of the corresponding parts of the brain increased.

The structure of the brain has been formed in the course of evolution. Evolution of the species occurs as a result of mutations during the process of appearance, better survival and expansion of individual species organisms because of their competitiveness. That is, the evolution of the species occurs through small discrete changes at the birth of each new organism and fixation of the features by means of a faster expansion of well-formed organisms that have won competition in the existing environment. The same properties can be either winning or losing under different conditions. For example, the presence of a warm cover gives advantages to animals living in cold regions of the Earth. At the same time, such animals cannot exist in conditions of a warm climate.

\section{Conclusion}

Analysis of functioning of the natural memory makes clear that there is no special mechanism, which analyzes the events occurred and recorded in a short-term memory, and transfers the most important of these events to a long-term memory. All this would be too difficult even in the case (with a low probability of achieving), if scientists and engineers could have created such a mechanism, whereas natural information processing systems are formed spontaneously in each organism as a result of the self-organization of elements (neurons and connections between them) that form the structure of the brain. 
Our analysis shows that the paths of signal (information) propagation in the brain parallelize and fall into different areas of the brain, where they are fixed. In these zones, as a rule, information is recorded that relates primarily to a certain group of receptors and to a certain activity. Here, the word "primarily" means "mostly", but not "exclusively".

Therefore, associations between events of various kinds can be fixed, and reliability is ensured; if a part of the brain is damaged information is usually lost not completely because of the dispersal of the places of fixation of information, although reproduction can be difficult.

Since traces of events along the way are fixed in a variety of places including zones with different rates of forgetting, then after a while short-term memory fades so much that the information recorded in it can be considered lost irrevocably. As a result, when you try to remember some episodes, information about events recorded in areas with a lower forgetting rate comes to the fore, but already with several other associations than information in a short-term memory. Therefore, researchers at different levels have the impression that the processed information is moving into a long-term memory. It seems to be recycled (undergoing processing) because the information fixed in a long-term memory comes to the fore. Information in a long-term memory (with a lower rate of extinction) is connected with associations that differ from associations that are inherent in information in a short-term memory.

Relocation of information from a short-term memory to a long-term memory is absent. Just a short-term memory about the event (with its associations) is thrown into the background.

If nature, as a result of evolution, has found it expedient to have zones in the brain with different rate of memorization and memory fading, then it is probably expedient to create such zones in the memory of artificial devices of the intelligence carriers. It is clear that nature does not have any goals (processes in it simply proceed according to its laws), so we should talk not about expediency, but about mutual consistency of processes. Forgetting is useful, it provides variability of the organism's response.

\section{Acknowledgements}

The research presented in this paper was supported by the Russian Foundation for Basic Research (grant No 16-29-04388/17). The authors are grateful to Tatiana B. Rumyantseva from Tomsk State University for English language editing.

\section{References}

[1] V. N. Shumilov Principles of the Brain Functioning. Engineer's look. (without PH Ekaterinburg, 2008)

[2] Psychology. Features of Memory as a Psychological Process URL: $\quad$ http://psixologiya.org/obshhaya/pamyat/1647-osobennosti-pamyati-kakpsixologicheskogo-proczessa-diplom.html

[3] V. N. Lange About the Rate of Forgetting URL: http://www.voppsy.ru/issues/1983/834/834142.htm

[4] Evolution of the Animals' Brain http://biofile.ru/bio/19130.html

[5] Biology and medicine. Evolution of the Brain in Vertebrates URL: http://medbiol.ru/medbiol/antrop/00079dc7.htm

[6] V. N. Shumilov, I. V. Shumilov, V. I. Syryamkin, Neurocomputers: Development and Application 4, 94(2015) 
[7] V. N. Voronin, V. N. Shumilov, Yu. A. Shumilov, A. R. Tsoy, A.V. Kiselev, S. M. Sharshov, Neurocomputers: Development and Application 4, 26 (2015)

[8] Short-term Memory URL: https://ru.wikipedia.org/wiki/\%D0\%9A\%D1\%80\%D0\%B0\%D1\%82\%D0\%BA $\%$ D0 $\% \mathrm{BE} \% \mathrm{D} 0 \% \mathrm{~B} 2 \% \mathrm{D} 1 \% 80 \% \mathrm{D} 0 \% \mathrm{~B} 5 \% \mathrm{D} 0 \% \mathrm{BC} \% \mathrm{D} 0 \% \mathrm{~B} 5 \% \mathrm{D} 0 \% \mathrm{BD} \% \mathrm{D} 0 \% \mathrm{BD} \% \mathrm{D} 0 \% \mathrm{~B}$ $0 \% \mathrm{D} 1 \% 8 \mathrm{~F} \_\mathrm{D} 0 \% \mathrm{BF} \% \mathrm{D} 0 \% \mathrm{~B} 0 \% \mathrm{D} 0 \% \mathrm{BC} \% \mathrm{D} 1 \% 8 \mathrm{~F} \% \mathrm{D} 1 \% 82 \% \mathrm{D} 1 \% 8 \mathrm{C}$

[9] G. Ebbingauz "Psychology" (1907)

[10] G. A. Miller. The Psychological Review 63, 81 (1956) 